\title{
Research on Horizontal Stability Model of Six-Axis Simulation/Testing Turntable
}

\author{
J.H. Zhou \\ School of Information Engineering \\ Nanchang Hangkong University \\ China
}

\author{
X.F. Peng \\ College of Information \& Technology \\ Xiamen University \\ China
}

\begin{abstract}
In order to shorten the shipboard stable platform's development cycle, reduce its development cost and facilitate experimental research, a new type of six-axis simulation/testing turntable was proposed in the paper. The turntable's application and performance index were introduced from the system's composition and key performance indicators point of view. The tested equipment was installed in the inner frame plane of turntable's upper body, and the mathematic model which realizes horizontal stability of the inner frame plane of turntable's upper body was computed with coordinate transformation when the outer frame of turntable's upper body and the lower body of turntable rotate. A short conclusion is drawn by analysis of simulation results.
\end{abstract}

Keywords-simulation/test turntable; horizontal stability; mathematic model

\section{INTRODUCTION}

The products used on ship need to be tested repeatedly in the process of research and development for verifying the validity and feasibility of them. If each test is carried out to sea, it will take a lot of manpower, material and financial resources and time. In order to facilitate test research and save test cost, we design a new type six-axis simulation/testing turntable, which can simulate the ship attitude in various sea conditions [1-4]. So, it can provide technical support to research the new products of stable platform in the laboratory, reduce the test cost and shorten the development cycle. The paper mainly researches horizontal stability model of six-axis simulation/testing turntable, and its function was to keep the table which is installed the precision equipments parallel with the local level and achieve the stability of the precision equipments, so it can eliminate the impact of the rocking base.

Simulation/testing turntable is the key support equipment for semi-physical simulation and testing in the marine, aviation, aerospace and defence domain, so it plays an extremely important role in the development process of related equipments [5]. Simulation/testing turntable can realistically simulate attitude of vehicle in the actual space, and reproduce the dynamic characteristics in the runtime, and thus it can help designer improve the related equipments [6-8]. Furthermore, simulation/testing turntable can be used as a stable platform which can effectively isolate the motion of vehicle and ensure the attitude stabilization of equipments mounted on the turntable. Simulation/testing turntable can be used in just about every type of moving vehicle as well as some handheld and ground-mounted applications [9], such as Hubble Space Telescope which is equivalent to looking at a dime from $200 \mathrm{mi}$, military system which can use it to control the targeting, or tracking, system as well as the weapon aimpoint, the handheld camera and so on.

The rest of this paper is organized as follows. In Section 2, we present the structure and performance of six-axis simulation/testing turntable. Then the horizontal stability model of six-axis simulation/testing turntable is derived by coordinate transformation in Section 3. Thereafter, simulation and a short conclusion are drawn in Section 4.

\section{SIX-AXIS SIMULATION/TESTING TURNTABLE}

Six-axis simulation/testing turntable is mainly composed of machinery body, DC torque motor servo control system, angle measurement system and computer control system. Machinery body is composed of upper body and lower body. The lower body is a three-axis turntable which simulates the rocking motion of a ship on the sea, and the upper body is a three-axis turntable too which simulates stable and tracking motion of platform. They are both U-O-O structure type, which inner frame and middle frame are both O-type structure, and outer frame is U-type structure. The upper and lower body can be used alone or in combination. When they are used in combination, the upper body is mounted on the outer frame of the lower body, and the six-axis simulation/testing turntable is created, see fig. 1 . 


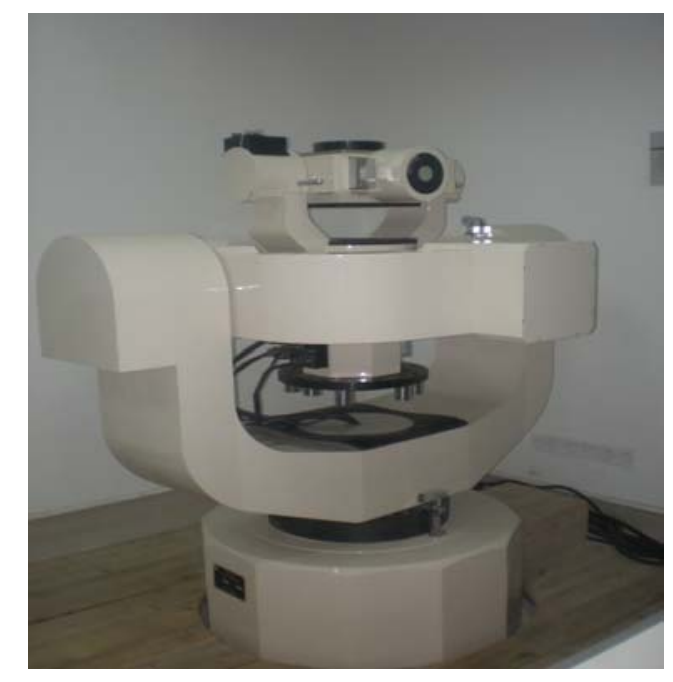

FIGURE I.SIX-AXIS SIMULATION/TESTING TURNTABLE.

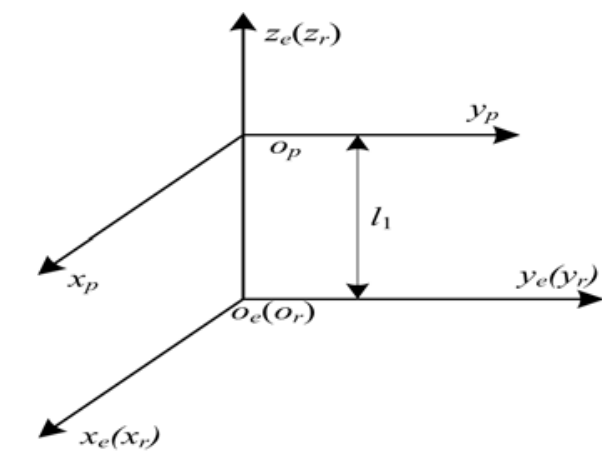

FIGURE II.

COORDINATE SYSTEMS.

The key performance indicators of six-axis simulation/testing turntable are mainly as follows: (1) When the lower body is used alone, the angle range of lower body's inner and middle frame are respectively [-60o 60o] and [-45o $45 \mathrm{o}]$, and the outer frame of lower body is consecutive rotation. When the upper body and lower body are used in combination, the angle range of lower body's inner frame and middle frame are respectively [-15o 15o] and [-15o 15o], and the outer frame of lower body is consecutive rotation. The angle range of upper body's inner frame and middle frame are respectively [-20o 20o] and [-20o 20o], and the outer frame of upper body is consecutive rotation. (2) Each frame positioning accuracy of the upper body and lower body is respectively better than $1^{\prime}$ and 0.020 . (3) The six-axis simulation/testing turntable has no creeping phenomenon and jitter when it is low speed, and its resolution is better than $0.01 \mathrm{o} / \mathrm{s}$. (4) The motion frequency of the upper body's inner frame and middle frame are also not less than $5 \mathrm{~Hz}$, and the motion frequency of the lower body's inner frame and middle frame are also not less than $5 \mathrm{~Hz}$. The close loop phase shift is not more than $10 \mathrm{o}$ and the amplitude bandwidth is not more than $10 \%$ when the motion frequency of the outer frame is not less than $3 \mathrm{~Hz}$. (5) The turntable has the functions of over-speed protection, overcurrent protection and emergency power-off protection. Furthermore, the turntable can stop at the current position and not produce violent jitter when it encounters the emergency power-off. (6) The upper body can carry the load of $8 \mathrm{Kg}$ or less, and the steady and stable working time of six-axis simulation/testing turntable is not less than 8 hours.

\section{HORIZONTAL STABILITY MODEL}

The inner frame, middle frame and outer frame of upper body are respectively parallel to the inner frame, middle frame and outer frame when the six-axis simulation/testing turntable is in the initial attitude, and the initial attitude of upper body and lower body are also parallel to ground plane. The tested equipment is mounted on the plane of the upper body's inner frame. The outer frame motion, middle frame motion and inner frame motion of lower body respectively simulate the ship movement of yaw, pitch and roll. The outer frame motion of upper body simulates tracking angle of tested equipment. The horizontal stability of tested equipment is achieved by middle frame motion and inner frame motion of upper body.

To facilitate the derivation of the horizontal stability model, we create the following three coordinate systems, i.e. geographic coordinates, turntable coordinates and platform coordinates, and they are shown in fig. 2. The origin of geographic coordinates i.e. oe-xeyeze is established at the intersection point of the lower body's three frames, the axis oexe, axis oeye and axis oeze respectively point to east direction, north direction and zenith direction of ground. The initial position of turntable coordinates i.e. or-xryrzr fully coincides with the geographic coordinates, and it rotates with the lower body. Furthermore, the axis orxr, axis oryr and axis orzr respectively coincide with the inner frame axis, middle frame axis and outer frame axis of lower body. The origin of platform coordinates i.e. op-xpypzp is established at the intersection point of the upper body's three frames. Furthermore, the axis opxp, axis opyp and axis opzp respectively coincide with the inner frame axis, middle frame axis and outer frame axis of upper body, and the platform coordinates rotates with the upper body.

The outer frame motion, middle frame motion and inner frame motion of lower body respectively simulate the ship movement of yaw, pitch and roll, set the yawing angle, pitch angle and rolling angle are respectively as follows: $\phi, \theta, \psi$. We convention sine function sin abbreviated as $\mathrm{S}$ and cosine function cos abbreviated as $\mathrm{C}$ in the paper. According to the generalized coordinates transformation, we can get the transformation formula between turntable coordinates and geographic coordinates shown as follows:

$$
\left[\begin{array}{c}
x_{e} \\
y_{e} \\
z_{e} \\
1
\end{array}\right]=T_{e r}\left[\begin{array}{c}
x_{r} \\
y_{r} \\
z_{r} \\
1
\end{array}\right]
$$

Where

$T_{e r}=\left[\begin{array}{cccc}\mathrm{C} \phi \cdot \mathrm{C} \theta & \mathrm{C} \phi \cdot \mathrm{S} \theta \cdot \mathrm{S} \psi-\mathrm{S} \phi \cdot \mathrm{C} \psi & \mathrm{C} \phi \cdot \mathrm{S} \theta \cdot \mathrm{C} \psi+\mathrm{S} \phi \cdot \mathrm{S} \psi & 0 \\ \mathrm{~S} \phi \cdot \mathrm{C} \theta & \mathrm{S} \phi \cdot \mathrm{S} \theta \cdot \mathrm{S} \psi+\mathrm{C} \phi \cdot \mathrm{C} \psi & \mathrm{S} \phi \cdot \mathrm{S} \theta \cdot \mathrm{C} \psi-\mathrm{C} \phi \cdot \mathrm{S} \psi & 0 \\ -\mathrm{S} \theta & \mathrm{C} \theta \cdot \mathrm{S} \psi & \mathrm{C} \theta \cdot \mathrm{C} \psi & 0 \\ 0 & 0 & 0 & 1\end{array}\right]$ (2) 
The coordinate of platform coordinates origin in turntable coordinates is $\left(\begin{array}{lll}0 & 0 & l_{1}\end{array}\right)$, the outer frame angle, middle frame angle and inner frame angle of upper body are respectively $\gamma$, $\beta$, and $\alpha$. According to the generalized coordinates transformation, we can get the transformation formula between platform coordinates and turntable coordinates shown as follows:

$$
\left[\begin{array}{c}
x_{r} \\
y_{r} \\
z_{r} \\
1
\end{array}\right]=T_{r p}\left[\begin{array}{c}
x_{p} \\
y_{p} \\
z_{p} \\
1
\end{array}\right]
$$

Where

$$
T_{r p}=\left[\begin{array}{cccc}
\mathrm{C} \gamma \cdot \mathrm{C} \beta & \mathrm{C} \gamma \cdot \mathrm{S} \beta \cdot \mathrm{S} \alpha-\mathrm{S} \gamma \cdot \mathrm{C} \alpha & \mathrm{C} \gamma \cdot \mathrm{S} \beta \cdot \mathrm{C} \alpha+\mathrm{S} \gamma \cdot \mathrm{S} \alpha & 0 \\
\mathrm{~S} \gamma \cdot \mathrm{C} \beta & \mathrm{S} \gamma \cdot \mathrm{S} \beta \cdot \mathrm{S} \alpha+\mathrm{C} \gamma \cdot \mathrm{C} \alpha & \mathrm{S} \gamma \cdot \mathrm{S} \beta \cdot \mathrm{C} \alpha-\mathrm{C} \gamma \cdot \mathrm{S} \alpha & 0 \\
-\mathrm{S} \beta & \mathrm{C} \beta \cdot \mathrm{S} \alpha & \mathrm{C} \beta \cdot \mathrm{C} \alpha & l_{1} \\
0 & 0 & 0 & 1
\end{array}\right] \text { (4) }
$$

Take three points on inner frame plane of upper body

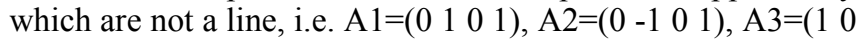
0 1). After rotation, the coordinate of $A 1, A 2$ and $A 3$, in geographic coordinates are respectively as follows:

$$
\left\{\begin{array}{l}
A_{1 e}=T_{e r} \cdot T_{r p} \cdot A_{1} \\
A_{2 e}=T_{e r} \cdot T_{r p} \cdot A_{2} \\
A_{3 e}=T_{e r} \cdot T_{r p} \cdot A_{3}
\end{array}\right.
$$

The axis oeze coordinate component in geographic coordinates of $\mathrm{A} 1, \mathrm{~A} 2$ and $\mathrm{A} 3$ can be given as the following equation by substituting the coordinate of $\mathrm{A} 1, \mathrm{~A} 2$ and $\mathrm{A} 3$, eqn. (2) and eqn. (4) into eqn. (5):

$$
\left\{\begin{array}{rl}
z_{A_{A_{e}}}= & -\mathrm{S} \theta \cdot(\mathrm{C} \gamma \cdot \mathrm{S} \beta \cdot \mathrm{S} \alpha-\mathrm{S} \gamma \cdot \mathrm{C} \alpha)+\mathrm{C} \theta \cdot \mathrm{S} \psi \cdot(\mathrm{S} \gamma \cdot \mathrm{S} \beta \cdot \mathrm{S} \alpha+\mathrm{C} \gamma \cdot \mathrm{C} \alpha) \\
& +\mathrm{C} \theta \cdot \mathrm{C} \psi \cdot\left(l_{1}+\mathrm{C} \beta \cdot \mathrm{S} \alpha\right) \\
z_{A_{A_{2 e}}} & \mathrm{~S} \theta \cdot(\mathrm{C} \gamma \cdot \mathrm{S} \beta \cdot \mathrm{S} \alpha-\mathrm{S} \gamma \cdot \mathrm{C} \alpha)-\mathrm{C} \theta \cdot \mathrm{S} \psi \cdot(\mathrm{S} \gamma \cdot \mathrm{S} \beta \cdot \mathrm{S} \alpha+\mathrm{C} \gamma \cdot \mathrm{C} \alpha) \\
& +\mathrm{C} \theta \cdot \mathrm{C} \psi \cdot\left(l_{1}-\mathrm{C} \beta \cdot \mathrm{S} \alpha\right) \\
z_{A_{A_{e}}}= & -\mathrm{S} \theta \cdot \mathrm{C} \gamma \cdot \mathrm{C} \beta+\mathrm{C} \theta \cdot \mathrm{S} \psi \cdot \mathrm{S} \gamma \cdot \mathrm{C} \beta+\mathrm{C} \theta \cdot \mathrm{C} \psi \cdot\left(l_{1}-\mathrm{S} \beta\right)
\end{array}\right.
$$

If the inner frame plane of upper body is parallel to the ground plane, the axis oeze coordinate component in geographic coordinates of A1, A2 and A3 must satisfy the following equation:

$$
\left\{\begin{array}{l}
z_{A_{1 e}}=z_{A_{2 e}} \\
z_{A_{2 e}}=z_{A_{3 e}}
\end{array}\right.
$$

Angle $^{\phi}, \theta, \psi$ and $\gamma$ are known quantity, which were obtained by the sensors. Then we can get the following equation by substituting the eqn. (6) into eqn. (7):

$$
\begin{aligned}
& \mathrm{S} \theta \cdot(\mathrm{C} \gamma \cdot \mathrm{S} \beta \cdot \mathrm{S} \alpha-\mathrm{S} \gamma \cdot \mathrm{C} \alpha)-\mathrm{C} \theta \cdot \mathrm{S} \psi \cdot(\mathrm{S} \gamma \cdot \mathrm{S} \beta \cdot \mathrm{S} \alpha+\mathrm{C} \gamma \cdot \mathrm{C} \alpha) \\
& -\mathrm{C} \theta \cdot \mathrm{C} \psi \cdot \mathrm{C} \beta \cdot \mathrm{S} \alpha=0 \\
& \mathrm{~S} \theta \cdot(\mathrm{C} \gamma \cdot \mathrm{S} \beta \cdot \mathrm{S} \alpha-\mathrm{S} \gamma \cdot \mathrm{C} \alpha)-\mathrm{C} \theta \cdot \mathrm{S} \psi \cdot(\mathrm{S} \gamma \cdot \mathrm{S} \beta \cdot \mathrm{S} \alpha+\mathrm{C} \gamma \cdot \mathrm{C} \alpha) \\
& -\mathrm{C} \theta \cdot \mathrm{C} \psi \cdot \mathrm{C} \beta \cdot \mathrm{S} \alpha+\mathrm{S} \theta \cdot \mathrm{C} \gamma \cdot \mathrm{C} \beta-\mathrm{C} \theta \cdot \mathrm{S} \psi \cdot \mathrm{S} \gamma \cdot \mathrm{C} \beta+\mathrm{C} \theta \cdot \mathrm{C} \psi \cdot \mathrm{S} \beta=0 \\
& \quad \mathrm{Substitute} \mathrm{the} \mathrm{eqn.} \mathrm{(8)} \mathrm{into} \mathrm{eqn.} \mathrm{(9),} \mathrm{we} \mathrm{can} \mathrm{obtain:}
\end{aligned}
$$

$$
(\mathrm{S} \theta \cdot \mathrm{C} \gamma-\mathrm{C} \theta \cdot \mathrm{S} \psi \cdot \mathrm{S} \gamma) \cdot \mathrm{C} \beta+(\mathrm{C} \theta \cdot \mathrm{C} \psi) \cdot \mathrm{S} \beta=0(10)
$$

Then the angle $\beta$ can be obtained by eqn. (10):

$$
\beta=\arctan \left(\frac{\mathrm{S} \theta \cdot \mathrm{C} \gamma-\mathrm{C} \theta \cdot \mathrm{S} \psi \cdot \mathrm{S} \gamma}{\mathrm{C} \theta \cdot \mathrm{C} \psi}\right)
$$

Substitute the angle $\beta$ into eqn. (8), we can obtain:

$$
\begin{aligned}
& (\mathrm{S} \theta \cdot \mathrm{C} \gamma \cdot \mathrm{S} \beta-\mathrm{C} \theta \cdot \mathrm{S} \psi \cdot \mathrm{S} \gamma \cdot \mathrm{S} \beta-\mathrm{C} \theta \cdot \mathrm{C} \psi \cdot \mathrm{C} \beta) \cdot \mathrm{S} \alpha \\
& =(\mathrm{S} \theta \cdot \mathrm{S} \gamma+\mathrm{C} \theta \cdot \mathrm{S} \psi \cdot \mathrm{C} \gamma) \cdot \mathrm{C} \alpha
\end{aligned}
$$

Then the angle $\alpha$ can be obtained by eqn. (2):

$$
\alpha=\arctan \left(\frac{\mathrm{S} \theta \cdot \mathrm{S} \gamma+\mathrm{C} \theta \cdot \mathrm{S} \psi \cdot \mathrm{C} \gamma}{\mathrm{S} \theta \cdot \mathrm{C} \gamma \cdot \mathrm{S} \beta-\mathrm{C} \theta \cdot \mathrm{S} \psi \cdot \mathrm{S} \gamma \cdot \mathrm{S} \beta-\mathrm{C} \theta \cdot \mathrm{C} \psi \cdot \mathrm{C} \beta}\right)
$$

Therefore, the eqn. (11) and eqn. (13) together form the horizontal stability model of six-axis simulation/testing turntable.

\section{SIMULATION AND CONCLUSIONS}

Simulation studies were conducted on Matlab. The simulations were carried out with yawing angle $\phi=\pi / 8 \sin 0.628 t$, pitch angle $\theta=\pi / 12 \sin 0.628 t$, rolling angle $\psi=\pi / 15 \sin 0.628 t$ and the outer frame angle of upper body $\gamma=15 \mathrm{o} / \mathrm{s}$, then they are shown in fig. 3. Then simulation studies were conducted in accordance with the horizontal stability model, and we can obtain the results shown in fig. 4.

In this paper a simple approach to solve the horizontal stability model of six-axis simulation/testing turntable. The horizontal stability model does not matter with the rotation angle of lower body's outer frame, because the inner frame of lower body, the middle frame of lower body and the upper body rotate together with the outer frame of lower body. In the horizontal stability tracking process, the amplitude and phase of compensation angle will change with the rotation angle of upper body's outer frame.
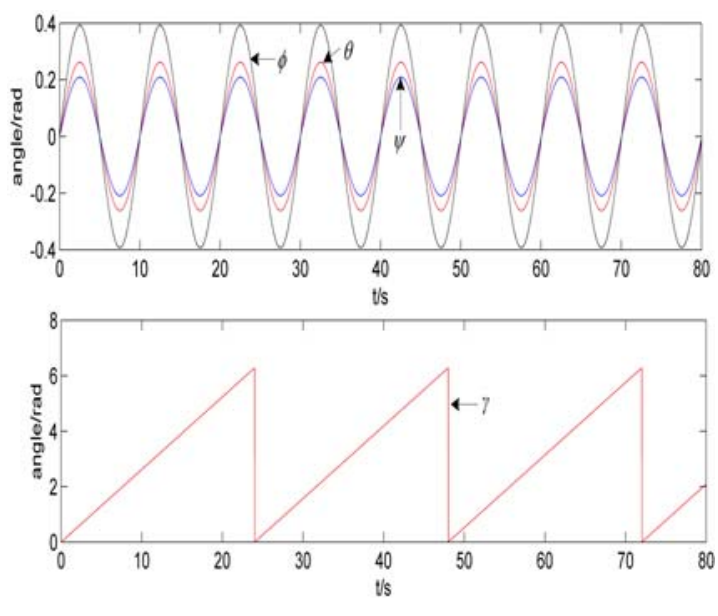

FIGURE III. INPUT SIMULATION ANGLE. 


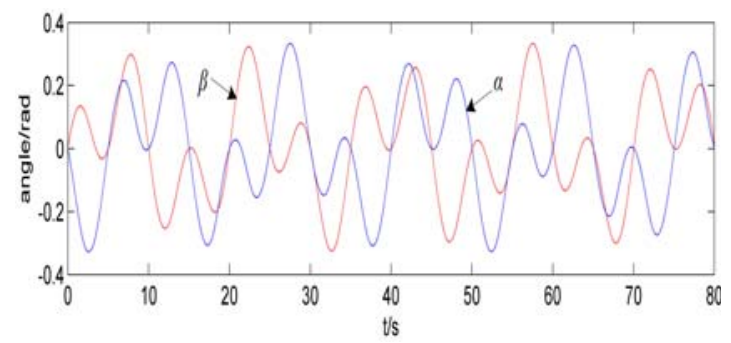

FIGURE IV. COMPENSATION ANGLE.

\section{ACKNOWLEDGMENT}

The authors would like to express financial supports from Foundation Project of Jiangxi Provincial Department of Education (GJJ14549), Nature Science Foundation of Fujian Province (2010J05141) and Doctor Scientific Research Foundation of Nanchang Hangkong University (EA201204428).

\section{REFERENCES}

[1] Jin Hongzhang, Guo Jian \& Chen Fang, Mathematica model of TACAN antenna stable platform of naval vessels, Ship Building of China, 43(4), pp.55-62, 2002.

[2] Deng Zhihong \& Liu Sheng, Setting-up of mathematic platform and simulation of shipborns strap-down minehunting sonar array, Ship Engineering, 23(1), pp.50-64, 2001.

[3] Meng Hao, Zhao Guoliang \& Wang Lan, The hybrid simulation system for ship's motion, Journal of System Simulation, 15(3), pp. 457-459, 2003 .

[4] Zhou Jiehua, Peng Xiafu \& Zhong Xunyu, Six-axis ship swaying simulation rotationtable and orientation stable tracking model. Ship Engineering, 33(2), pp.53-57, 2011.

[5] Zhao Yuhua, Yuan Feng \& Ding Zhenliang, Error modeling and analysis of three-axis simulation turntable. Journal of Sichuan University (Engineering Science Edition), 42(6), pp. 227-231, 2010.

[6] Wang Xiaojun, Li Dianpu, Zao Yan, Yu Hongming \& Zheng Jinyong, 3 -axis steady-tracking with shipborne radar, Journal of Harbin Engineering University, 23(1), pp. 58-63, 2002.

[7] Liu Chaojun, Liao Xiaozhong \& Zhang Yuhe, Kinematics modeling and simulation of strapdown 3 -axis stable tracking system, Journal of Beijing Institute of Technology, 20(3), pp. 313-316, 2000.

[8] Fan Dapeng, Zhang Zhiyong, Fan Shixun \& Li Yan, Research of basic principles of E-O stabilization and tracking devices, Optics and Precision Engineering, 14(4), pp. 673-678, 2006.

[9] Hilkert J. M., Inertially stabilized platform technology. IEEE Control Systems Magazine, 28(1), pp. 26-46, 2008. 